\title{
TOWARDS A THEORY OF LINGUISTIC MEMORY
}

\author{
Terrence J. Keeney \\ University of California, Riverside
}

Every day we are exposed to a vast amount of linguistic material. We hear the speech of others; we read signs, newspapers, books, etc.; and we hear our own speech. Although we do not attend to and fully process all of this linguistic material, there remains a substantial sum of it which we do pay attention to, process, and comprehend. Yet, of all this comprehended material, we know from our own personal experience that we can recall verbatim only a tiny portion. When accurate verbatim recall becomes important, for example, in courtroom testimony, the fallibility of even this tiny portion becomes woefully apparent. Rarely can we accurately recall even what we ourselves have said previously.

It is this memory for what we have heard or read that I am referring to by the term "linguistic memory." Linguistic memory must not be confused with semantic memory, the topic of the papers by Loftus and Glass in this conference. Semantic memory refers to the "permanent" storage of morphemes or concepts in the human or artificial brain. Linguistic memory, on the other hand, refers to the reproduction or recognition of prior linguistic inputs. As we shall see, linguistic memory draws upon semantic memory, but it includes, in addition, information about specific, time-dependent, prior events.

By analogy to mechanical devices that have been designed for preserving speech and writing, we might consider linguistic memory as a process which preserves the physical features of the linguistic input. Just as a photocopy or a tape recording encode the electromagnetic and acoustic features of writing and speech, preserve them for some time, and then reproduce or play back the oxiginal physical features with some degree of approximation to the original, so the human sensory and central nervous processes would encode the physical features of the input, store these features, and then reproduce them at some later time through the appropriate neuro-muscular commands.

In order to account for our very limited ability to accurately recall the prior inputs, we would need to introduce inefficient encoding and decoding devices and a rapidly deteriorating storage or a storage which is easily disrupted by interference from prior and subsequent inputs. Physical analogies to these processes could be constructed by the use of very grainy emulsions for a photocpy or low-fidelity magnetic tape for a sound recording, copies that fade or recording tape that disintegrates over time, the superimposition of photos or tapes, and inefficient read-out or playback equipment.

The critical feature of all such devices, despite the differential accuracy of their reproductions, is the fact that they operate on the physical features of the input. Any loss 
of information or errors in recall must therefore be related to the physical features of the input. For heard messages, phonemes that sound alike would be substituted for one another and pauses and intonations would be lost or distorted. For read messages, the same types of errors would occur and, in addition, letters that looked alike might be substituted for one another and punctuation marks and spacings would be lost or distorted. From studies of auditory and visual perception and short-term memory for isolated phonemes and letters (Wicklegren, 1966; Gibson, et al, 1962), we know enough about the relevant visual and auditory analyzers to be able to predict the nature of these types of errors in linguistic memory.

However, when we investigate the nature of the errors made in both the recall and recognition of coherent linguistic material, we find that the model described above which is based on the physical features of the input fails. Bartlett (1932) studied the recall of short stories and found regularities within the errors committed; but these errors had to do with the "sharpening" and "leveling" of themes and ideas, not with the confusion of similar sounds or letters. Fillenbaum (1966) investigated mamory for negative constructions and found accurate memory of the "gist" of what had been read, but not of the specific words or syntactic constructions.

Sachs (1967a) studied memory for connected discourse by introducing recognition-test sentences at the conclusion of short stories. When these test sentences were active-topassive or "formal" transforms of original story sentences, her subjects gave little evidence that they could detect any difference between the original and test sentences, even though they were explicitly instructed to try and detect changes in form as well as in meaning. On the other hand, transforms of an original story sentence which reverse the actor-object relation or in some other way drastically altered the meaning of a story sentence were readily detected as being different. These meaning changes were detected despite the fact that they did not alter the word order or actual form of the sentence any more than did the undetectable activeto-passive and "formal" transforms.

In a subsequent study (Sachs, 1967b), she found that change even in the actual words of a sentence introduced by the substitution of synonyms in the recognition test was generally not detected. Furthermore, the active-to-passive, "formal", and synonym-substitution transforms were undetectable when there was as little as 7.5 seconds of filled (with further story material) time elapsing between the sentence and its recognition test.

Mathewson and Keeney (1971) modified the Sachs' procedure so that meaning and form could be varied completely independently of one another. By the use of deep-structure ambiguous sentences such as, "He was in fact the one to hear before leaving" "we were able to change the meaning of a sentence without introducing any change in the form of the sentence itself. This was accomplished by varying the linguistic context in which the sentence was 
embedded. In the example below, the meaning of the sentence changes from the story to the test simply as a result of its context.

\section{Story}

The speaker from New York was by far the best. He spoke about the problems of deaf education. He brought forth some very interesting ideas. The ideas concerned the social and personal problems in educating deaf people. None of the other speakers impressed me as much. The one I mentioned was the only one worth lisiening to. He was, in fact, the one to hear before leaving.

\section{Test}

Of all the people who underwent surgery for deafness, only Bill left the hospital happy. He was, in fact, the one to hear before leaving.

The findings of this study confirmed and extended those of Sachs'. Meaning changes, even when they involved no change in the form of the sentence, were readily detected. Form changes such as article or adverb movement transforms, e.g. "He was, in fact, the one to hear before leaving. /In fact, he was the one to hear before leaving", which preserved the meaning, were not detected even when as few as 25 syllables of material intervened between the sentences, as in the example above. In this study, as well as in those of Sachs, these results cannot be ascribed to the subjects' lack of awareness of the types of changes to be detected. Prior to the experiment the subjects were instructed to detect both formal change and meaning change. In addition, they were shown examples of each type of change. In order to produce such rapid forgetting of the purely formal features of linguistic material, it is necessary only that the subject be listening or reading normally and comprehending the material rather than memorizing or rehearsing the sentences. Since it is impossible for most people to memorize sentences as rapidly as they are heard in normal speech, such rapid forgetting is the common experience. In the experiments cited above, the subjects did not know which of the sentences in the story they would subsequently be presented in the recognition test. Although instructed to remember form, they were unable to do so.

A recent experiment (Jarvella and Herman, 1972) suggests that the process of comprehension may, itself, contribute to, or at least allow for, the deterioration of verbatim memory. They investigated the running memory span for connected discourse. When recall was called for at the conclusion of a complex sentence, vexbatim recall of the first clause in the sentence was better for sentences of subordinate clause-mainclause construction than for their converse. If we make the reasonable assumption that a subordinate clause is not fully processed and thus not fully comprehended until its main clause has been heard and comprehended, we see that in the sentence in which the subordinate clause preceeds the main clause the subordinate clause must be held in some partially processed state until the main clause is understood. A main clause can, of course, be comprehended in isolation. Thus, we see that 
at the conclusion of the entire sentence the first clause in a subordinate-main sentence is still only partially comprehended, while the first clause in a main-subordinate sentence has been fully comprehended for some time. Therefore, the poorer verbatim recall in the mainsubordinate sentence suggests that comprehension of a clause actually diminishes the listeners' ability to recall that clause verbatim.

When exact verbatim recall is required, as in remembering songs, poems, rituals, etc., in which the form of expression is as important as the meaning, the special mnemonic devices of rhyme and/or rhythm are almost invariably present in the material itself. These devices provide a definite formal structure into which the meaning must be fitted. There may be many ways of expression a given idea, yet only one way which possesses the appropriate meter and rhyme. Thus memory for the meaning combined with the restrictions put on the form by the rhyme and rhythm result in accurate verbatim reconstruction of the original message without any actual verbatim storage of that message. The pervasiveness of meter and rhyme in the myths and unwritten histories of preliterate cultures belies the fact that verbatim recall without these structural restrictions is difficult, if not impossible.

A model of linguistic memory which consists of a copy of the surface physical features of the linguistic input is clearly inadequate. The individual sounds, morphemes, and syntactic structures are not directly stored in memory; nor does the input simply excite certain pre-existing morpheme "nodes" in a permanent memory storage system. Rather, linguistic memory depends on an analysis of the meaning of the input.

That meaning and not form is the primary information retained in the memory of words embedded within sentences was demonstrated by Bobrow (1970). He selected a number of ambiguous nouns and presented them as subject and object nouns of sentences. The meanings of these ambiguous nouns were determined by the context established by the sentence in which they were embedded. An example of two sentences in which the nouns take on different meanings is "The pine board shored the river bank. / The securities board closed the shaky bank. "

A list of sentences was presented to the subjects to study for later recall. Each noun pair was presented twice in the course of this list. These noun pairs were repeated in one of three ways: I) exact sentence repetition, II) change in the sentence context which maintained the same noun meanings, III) change in the sentence context which changed the noun meanings. On the recall test the subject-noun was given as a cue for the recall of the objectnoun.

If the actual physical features of the input were remembered, then the recall in all three conditions of repetition should have been equal, since the nouns were presented twice in all conditions. However, if the meaning of the input was remembered, then recall in 
Condition I and II should have been superior to recall in Condition III. The meaning of the nouns changed from the first to the second presentation of the sentence in Condition III; so, in effect, the word meanings were not repeated. Thus, by virtue of their repetition within the list, the word meanings in Conditions $I$ and II should have been recalled with a greater frequency than the once-presented meanings of Condition III.

Bobrow's results confirmed the meaning-based memory model and were opposed to the form-based memory model. Recall in Conditions I and II was equal and superior to recall in Condition III. Changing the meaning of a word when it is repeated does not increase the probability of its recall as much as if it were repeated with the same meaning.

Not only are the forms of morphemes, words, and sentences not retained exactly in memory, even the division between sentences is not retained. Bransford and Franks (1971) presented sentences that contained one, two, or three of a total of four related "linguistic ideas". In a subsequent recognition test in which the subjects were asked to indicate whether or not they had actually heard the exact sentence before, the sentences with all four of the related ideas were most often "recognized" as having been heard before, even though, in reality, they had not been previously presented. In linguistic memory, then, the separation between sentences is not retained and ideas which "go together", but which are heard in separate sentences, are combined.

The evidence that we have reviewed up to this point is consonant with a model of memory in which not the form of the linguistic input, but some other aspect of it, which we have called "meaning", is encoded, stored, and retrieved. However, even this meaningbased model is inadequate, for we "remember" meanings that we have never before heard explicitly stated. There is evidence to suggest that we cannot distinguish our inferences from our perceptual inputs.

Bransford et al.(1972) have demonstrated false recognition of sentences which contain information that was not explicitly stated in the initial input sentences. Rather, these falsely recognized sentences expressed meanings which could be inferred from a combination of the previous linguistic input and the listeners' knowledge of three-dimensional spatial relations. For example, the sentence "Three turtles rested on the floating $\log$ and a fish swam beneath them.", along with spatial knowledge, leads to the inference that the sentence "Three turtles rested on the floating $\log$ and a fish swam beneath it." is also true.

In a series of experiments, Bransford et al. presented sentences such as the one with "them" above and found that in a subsequent recognition test, in which the subjects were to indicate which sentences they actually had heard before, false recognition of the inferred sentences, such as the one with "it" above, was just as frequent as the correct recognition of 
the sentences actually heard. The subjects responded exactly as if they had heard the sentences that were only inferences from what they had heard! Control sentences that were only one-word different from the presented sentences, but were not inferences from them, were not recognized as having been heard before; so the above results are not due to the small formal difference between the presented and the inferred sentences. The results of recall tests were parallel to those of the recognition test. "The subjects "recalled" sentences which they had never heard before, but which were inferences from these previously heard sentences.

So, linguistic wemory does not involve simply an encoding or extraction of some information, i.e., meaning, which is different from the physical form of the input. Rather, linguistic memory is based on an active, constructive process which is triggered by some linguistic input, but is not restricted to that input. For lack of a better term, 1 will call this process understanding or thinking. The recalling or re-thinking of these initially triggered thoughts constitutes the phenomenon we call linguistic memory. When assessing the accuracy of memory we should seek isomorphism not between the perceptual input at time $\mathrm{T}_{0}$ and the subsequent organismic output at time $T_{1}$, but between the thought processes at times $T_{0}$ and $\mathrm{T}_{1}$

Any combination of linguistic and situational events which produces the same thoughts or leads to the same understanding should be indistinguishable in memory. For example, we would expect that all five of the following would trigger basically the same thoughts and would therefore all be confused one with the other in a recognition test.

1. "John had a favorite toy. He lost it. He was sad."

2. "John's favorite toy was lost, so he was sad."

3. "John had a favorite toy. He was sad because he lost it."

4. "John had a favorite toy. It was lost. That made John sad."

5. "John lost his favorite toy.", heard while looking at a picture of a boy with a sad expression on his face.

What combination of events activates a given thought is not remembered, rather, the thought process itself is repeated at the time of remembering. Not being able to remember whether we have read something or heard it is a common experience, and often we eventually discover that in fact we had neither read it nor heard it, but only thought it ourselves.

If the form of a linguistic input is very unusual, for example, if it is ungrammatical, poetic, employs strange metaphors, contains very low-frequency words, is spoken in an unfamiliar accent, etc., then certain thoughts may be activated by the form itself. Since thoughts are the "stuff" of memory, these thoughts about the form are capable of being rethought, i.e. remembered, at some later time giving the illusion that the form of the input 
has been retained. However, the thoughts about the unusual form are very rarely, if ever, isomorphic with the form of the input itself. For example, upon hearing an ungrammatical sentence, the meanings activated by the sentence and the fact that it is ungrammatical may be thought. Then, in a later recognition test, a grammatical sentence that activated the same thoughts about meaning would not be recognized because it did not activate the fact of ungrammaticality as did the initial input. However, any number of ungrammatical inputs which activated the appropriate thoughts would be confused with one another and all "recognized!" as having been heard before. The form itself is not remembered, only what is thought about it, i.e., that it is ungrammatical.

Once the focus is removed from the comparison of the perceptual input to the output as a measure of the accuracy of memory and placed instead on the comparison of the thought processes at times $\mathrm{T}_{0}$ and $\mathrm{T}_{1}$, much of the objectivity of research into memory phenomena is semmingly lost. The thoughts which are activated by linguistic and other events are not accessible to direct observation as are the events themselves. However, information about the nature of the relevant thought processes can be obtained by a comparison of those utterances which are all recognized as being what was heard previously. That is, the nature of the thoughts activated by an event are revealed by what is confused in a recognition test. Thus, if all five of the events described above are "recognized" as being what previously occurred, then we know that the information which distinguishes these events one from another is not part of the thought processes activated by their input.

It is reasonable to suppose that there will not be perfect isomorphism between the initial thought and the re-thought at the time of remembering. Recall or recognition will not always be perfect. The event that activates the re-thought will, itself, influence the nature of this re-thought. Furthermore, interference from other thoughts and, perhaps, some purely time-dependent decay process will alter the nature of the remembering. Assessments of the confusability or substitutability of a number of inputs at various times after the initial input will give an indication of these changes in memory.

One such change is a loss of differentiation or loss of specificity over time. Gary Olson (1971) investigated memory for prenominal adjectives heard in isolated sentences. In recognition tests he found false recognition for adjectives that were of the same general class as the adjectives actually heard in the initial sentences. For example, with the initial sentence, "A stone tower stood alongside the old building.", the incorrect adjective "wooden", was recognized more frequently than the incorrect adjectives, "round" and "square". The original understanding of the adjective, "stone", included the general notion of "type of mater ial". This general understanding or thought remained when the more specific information of the exact type of material was lost. It is in describing the nature of this understanding or 
thought process, not the nature of semantic memory, per se, that I believe the current work of Loftus and Glass in this conference will prove valuable.

The thoughts which are activated upon hearing a given Iinguistic input are related to the entire context in which they are heard. In fact, meaning itself depends on this context, as we have seen in the case of the ambiguous word or sentence. As David Olson (1970) has pointed out, the words of a message serve to differentiate some event from some set of alternatives. It is this differentiation process which is the meaning of the word.

I suggest that we remember only the degree of differentiation required by the context in which a message is heard. Take, for example, the sentence, "He ate the apple." When heard in the context of a description of a man at a smorgasbord choosing and eating various foods the meaning of this sentence will be remembered accurately. However, this very same sentence, when heard in the context of a story about a starving man who breaks into a house to get something to eat will be remembered at some time, $T_{1}$, as "He ate the fruil", and at some later time, $\mathrm{T}_{2}$, as "He ate the food." Despite this much loss of specificity over time, it will not continue the process and take on only the meaning, "He did something", because the context requires preservation of the information that the starving man ate something nourishing. The context of the smorgasbord story requires specification of the type of fruit; the context of the starving-man story requires specification only at the level of food. The though thoughts activated upon hearing any given word are fitted into the context of the entire message and, over time, these thoughts become only as specific as required by that context. Thus details often seem to "fade" in memory.

If it is the thoughts of the listener rather than the linguistic input itself which is the "content" of memory, then everything that affects thought affects memory. A whole host of organismic variables which are ignored in the input-based memory model become very important in the thought-based model. The listener actively constructs thoughts, and the external input is only one of the many determinants of these thoughts. Exactly the same input may well activate two very different thoughts in two different people. Consequently, their memories of what they have heard or seen will be very different. Although a model which incorporates the necessary organismic variables will necessarily be much more complex than the stimulusbased model, the attraction of the simpler model should not blind us to its inadequacies.

We have argued that, in some sense, linguistic memory does not exist. Rather, things heard or read contribute to the construction of internal thoughts in the listener. These thoughts are re-thought, with more or less fidelity, at the time of remembering. But it is the thoughts, not the linguistic input, which are remembered. 


\section{$\underline{\text { References }}$}

Bartlett, F.C. (1932), Remembering, Cambridge, England: Cambridge University Press.

Bobrow, S.A. (1970), "Memory for Words in Sentences", Journal of Verbal Learning and Verbal Behavior, $9,363-372$.

Bransford, J.D. and J.J. Franks (1971), "The abstraction of Linguistic Ideas", Cognitive Psychology, 2, 331-350.

Bransford, J.D., J.R. Barclay and J. J. Franks (1972), "Sentence Memory: Constructive versus Interpretive Approach", Cognitive Psychology, 3, 193-209.

Fillenbaum, S. (1966), "Memory for Gist: Some Relevant Variables", Language and Speech, 9, $217-227$.

Gibson, E.P., J.J. Gibson, A.D. Pick and H. Osser (1962), "A Developmental Study of the Discrimination of Letter-like Forms", Journal of Comparative and Physiological Psychology, 55, ง97-906.

Jarvella, R.J. and S.J. Herman (1972), "Clause Structure of Sentences and Speech Processing", Perception and Psychophysics, 11, 381-384.

Mathewson, G. C. and T.J. Keeney (1971), "Memory for Form and Meaning of Sentences Embedded in Paragraphs", Paper presented at the Western Psychological Association, San Francisco, April 22, 1971.

Olson, D.R. (1970), "Language and Thought: Aspects of a Cognitive Theory of Semantics", Psychological Review, 77, 257-273.

Olson, G. M. (1971), "Memory for Prenominal Adjectives in Ordinary English Sentences", Cognitive Psychology, $2,300-312$.

Sachs, J.S. (1967a), "Recognition Memory for Syntactic and Semantic Aspects of Connected Discourse", Perception and Psychophysics, 2, 437-442.

Sachs, J.S. (1967b), "Recognition of Semantic, Syntactic, and Lexical Changes in Sentences", Paper presented at Psychonomic Society, October, 1967.

Wicklegren, W.A. (1966), "Distinctive Features and Errors in Short-term Memory for English Consonants," Journal of the Acoustical Society of America, 39, 388-398. 